\title{
A New Model of Fuzzy Logic: Monadic Monoidal T- Norm Based Logic
}

Saeide Zahiri ( $\nabla$ saeede.zahiri@yahoo.com )

Shiraz University

Arsham Borumand Saeid

Shahid Bahonar University of Kerman

\section{Research Article}

Keywords: (Monadic) MTL-algebras, Monadic filter, Monadic monoidal t-norm logic.

Posted Date: January 4th, 2022

DOl: https://doi.org/10.21203/rs.3.rs-948685/v1

License: (c) (i) This work is licensed under a Creative Commons Attribution 4.0 International License. Read Full License 


\title{
A new model of fuzzy logic: Monadic Monoidal t-norm based logic
}

\author{
Saeide Zahiri $^{1 *}$, Arsham Borumand Saeid ${ }^{2}$ \\ 1 Department of Mathematics, Faculty of Science, Higher Education Center of Eghlid, Eghlid, Iran. \\ ${ }^{2}$ Department of Pure Mathematics, Faculty of Mathematics and Computer, Shahid Bahonar University of \\ Kerman, Kerman, Iran.
}

\begin{abstract}
In this article, we introduce the variety of monadic MTL-algebras as MTL-algebras equipped with two monadic operators. After a study of the basic properties of this variety, we define and investigate monadic filters in monadic MTL-algebras. By using the notion of monadic filters, we prove the subdirect representation theorem of monadic MTL-algebras and characterize simple and subdirectly irreducible monadic MTL-algebras. Moreover, present monadic monoidal t-norm based logic $(\mathcal{M M T} \mathcal{L})$, a system of many valued logic capturing the tautologies of monadic MTL-algebras and prove a completeness theorem.
\end{abstract}

Keywords: (Monadic) MTL-algebras, Monadic filter, Monadic monoidal t-norm logic.

AMS Classification: 08A72, 03G25, 03B50, $03 \mathrm{C} 05$.

\section{1. introduction}

Fuzzy logic has become a subject of increasing interest as the basis for reasoning with vague knowledge. In recent decades, different kinds of nonclassical logic systems have been proposed and researched. Among various non-classical logic systems, triangular norm and so monoidal tnorm based logic (MTL for short) is one of the most significant. The history of triangular norms started with the paper "Statistical metrics" (Menger 1942). The main idea of Karl Menger was to construct metric spaces where probability distributions rather than numbers are used in order to de scribe the distance between two elements of the space in question. Many results concerning t-norms were obtained in the course of this development, most of which are summarized in the monograph.

URL: saeede.zahiri@yahoo.com, s.zahiri@eghlid.ac.ir (Saeide Zahiri ${ }^{1} *$ ), arsham@uk.ac.ir (Arsham Borumand Saeid ${ }^{2}$ )

*Corresponding author. 
Building on these ideas, logicians like Hájek, Nóvak, Gottwald and others consider the core of fuzzy logic as a family of residuated many-valued logical calculi with truth values on the real unit interval $[0,1]$. Hájek introduced a very general many-valued logic, called Basic Logic [5]. It is a well-known result that a t-norm has a residuum if and only if the t-norm is left-continuous, so this shows that Basic Logic is not the most general t-norm based logic. Actually, a logic weaker than Basic Logic, called Monoidal t-norm based logic (MTL for short), was defined by Esteva and Godo in [4] and proved to be the logic of left-continuous t-norms and their residua. Thus, the MTL is indeed the logic of left-continuous t-norms, and MTL algebras are the algebraic counterpart of this logic. The point of departure in defining MTL and the corresponding MTL-algebras is the structure introduced on $[0,1]$ by a left continuous t-norm [4].

Halmos introduced monadic Boolean algebra: a variety of Boolean algebra equipped with a closure operator $\exists$, which abstracts algebraic properties of the standard existential quantifier for some [6]. The name monadic comes from the connection with predicate logics for languages having one placed predicates and a single quantifier. Similar algebraic structures were considered for various logics in $[7,8]$. Monadic Heyting algebras, monadic BL-algebras and monadic bounded residuated lattices were introduced and investigated in [10, 9]. J. T.Wang et al. introduced and considered monadic NM-algebras and their monadic filters and defined the relations between quantifiers and modal operators on NM-algebras [11].

The goal of this manuscript is to chart the landscape of formal fuzzy logics more specifically, to develop a logic that formally characterizes tautologies (true formulas) in similarity monadic MTL-algebras. To this purpose, in the present paper, we define and study monadic MTL-algebras endowed with unary operators $\forall$ and $\exists$, namely monadic MTL-algebras. In addition, monadic filters are defined. Definition of monadic filters in monadic MTL-algebras is different from that of filter in other algebraic structures such as MTL-algebras. Since operator $\forall$ and $\exists$ in this structure are important and are used in the definition of monadic filters, these types of filters play a basic role and thus the extended filters behave differently. And so, we give some properties of their factor algebras. In Section 2, we define the notion of monadic MTL-algebras and give their basic properties that will be used in the extant of the article. Therefore, the concept of monadic filters of monadic MTL-algebras have been introduced then, we classification these algebraic structure.

In this article, we will introduce the variety of monadic MTL-algebras and give a standard study of their basic properties, which includes the characterization of their congruences and subdirectly irreducible algebras. The notion of monadic filters on MTL-algebras have been defined and we 
consider them in detail. Since monadic filters enable the classification of monadic MTL-algebras, they are of particular importance. In particular, the paper [1] was a first attempt to define two

\section{Preliminaries}

We first recollect some definitions and results that are used later.

Definition 2.1. [4] $A$ residuated lattice is an algebra $\mathcal{A}=(A, \vee, \wedge, *, \rightarrow, 0,1)$ with four binary

about monadic MTL-algebras so for. It would be interesting to study monadic MTL-algebras in order to define and discuss monadic monoidal t-norm based logic. These are motivations for us to investigate monadic MTL-algebras and their logic.

In the first part of this paper, we overview recent results by the authors and others about properties that will be used in the extant of the article. Therefore, the concept of monadic filters of monadic MTL-algebras have been introduced then, by means of this notion we classification these algebraic structure. We define and characterize simple and subdirectly irreducible monadic MTLalgebras by monadic filters. In the last section, the monadic monoidal t-norm based $\operatorname{logic}(\mathcal{M M T} \mathcal{L})$ is defined, the Lindenbaum algebra of such logic, is a monadic MTL-algebra and completeness is proved. operations and two constant 0,1 such that:

$\left(R L_{1}\right)(A, \vee, \wedge, 0,1)$ is a bounded lattice, $\left(R L_{2}\right) *$ is commutative and associative, with 1 as neutral element, and $\left(R L_{3}\right) x * y \leq z$ iff $x \leq y \rightarrow z$, for all $x, y$ and $z$ in $A$ (residuation principle).

- A residuated lattice $A$ is called an MTL-algebra if it satisfies the following equation for all $a, b \in A:$

$(M T L)(x \rightarrow y) \vee(y \rightarrow x)=1$ (prelinearity).

- An MTL-algebra $A$ is called a BL-algebra if it satisfies the following equation for all $a, b \in A$ : $(B L) x \wedge y=x *(x \rightarrow y)$ (divisibility).

The ordering $\leq$ and negation $\neg$ in a residuated lattice $\mathcal{A}=(A, \vee, \wedge, *, \rightarrow, 0,1)$ are defined as follows, for all $x$ and $y$ in $A: x \leq y$ iff $x \wedge y=x$ (or equivalently, iff $x \vee y=y$; or, also equivalently, 
iff $x \rightarrow y=1)$ and $\neg x=x \rightarrow 0$.

Lemma 2.1. [4] Let $\mathcal{A}=(A, \vee, \wedge, *, \rightarrow, 0,1)$ be an $M T L$-algebra. Then the following properties are valid, for all $x, y$ and $z$ in $L$ :

(1) $\neg x \vee y \leq x \rightarrow y$,

(2) $x *(x \rightarrow y) \leq x \wedge y($ in particular $x * \neg x=0)$,

(3) $x \vee y \leq(x \rightarrow y) \rightarrow y($ in particular $x \leq \neg \neg x)$,

(4) $x \rightarrow y=((x \rightarrow y) \rightarrow y) \rightarrow y$,

(5) $\neg \neg \neg x=\neg x$,

(6) $(x \rightarrow y) * z \leq x \rightarrow(y * z)$,

80

(7) $(x \rightarrow y) *(y \rightarrow z) \leqslant(x \rightarrow z)$,

(8) $(x \vee y) \rightarrow z=(x \rightarrow z) \wedge(y \rightarrow z)($ in particular $\neg(x \vee y)=\neg x \wedge \neg y)$,

(9) $(x * y) \rightarrow z=x \rightarrow(y \rightarrow z)$ (in particular $\neg(x * y)=x \rightarrow \neg y)$,

(10) If $x \leqslant y$, then $x * z \leqslant y * z, z \rightarrow x \leqslant z \rightarrow y$ and $y \rightarrow z \leqslant x \rightarrow z$,

(11) $(y \rightarrow z) \leq(x \rightarrow y) \rightarrow(x \rightarrow z)$,

85

(12) $x \rightarrow(y \rightarrow z)=y \rightarrow(x \rightarrow z)$,

(13) $((x \rightarrow y) \rightarrow y) \rightarrow y=x \rightarrow y$,

(14) $x \rightarrow y \leqslant(y \rightarrow z) \rightarrow(x \rightarrow z)$,

(15) $\neg \neg x * \neg \neg y \leqslant \neg \neg(x * y)$.

From now on $(A, *, \rightarrow, \vee, \wedge, 0,1)$ or simply $A$ is an MTL-algebra unless otherwise specified.

90

Definition 2.2. [5] A deductive system of an $M T L$-algebra $A$ is a non empty subset $F$ containing 1 such that $x \rightarrow y \in F$ and $x \in F$ imply that $y \in F$.

A filter of an MTL-algebra $A$ is a non-void subset $F$ of $A$ such that, for all $a, b \in A$,

(i) if $x, y \in F$, then $x * y \in F$,

(ii) if $x \in F$ and $x \leq y$, then $y \in F$.

Note that a subset $F$ of an MTL-algebra $A$ is a deductive system of $A$ iff $F$ is a filter of $A$. 


\section{Monadic MTL-algebras and monadic filters}

In this section, we introduce monadic MTL-algebras and monadic filters on monadic MTLalgebras and investigate some of their algebraic properties. The relationship between monadic filters and some related structures will be discussed.

Definition 3.1. An algebra $(A, *, \rightarrow, \vee, \wedge, \exists, \forall, 0,1)$ is called a monadic MTL-algebra if $(A, *, \rightarrow$ $, \vee, \wedge, 0,1)$ is an $M T L$-algebra and the following identities are satisfied:

$\left(M_{1}\right) \forall x \rightarrow x=1$,

$\left(M_{2}\right) \forall(x \rightarrow \forall y)=\exists x \rightarrow \forall y$,

$\left(M_{3}\right) \forall(\forall x \rightarrow y)=\forall x \rightarrow \forall y$,

105

$\left(M_{4}\right) \forall(\exists x \vee y)=\exists x \vee \forall y$

$\left(M_{5}\right) \exists(x * x)=\exists x * \exists x$.

In the following we give some examples of monadic MTL-algebras.

Example 3.1. Let $A=\{0, a, b, c, 1\}$ and the operations $*, \rightarrow$ defined as follows:

\begin{tabular}{c|ccccc}
$*$ & 0 & $c$ & $a$ & $b$ & 1 \\
\hline 0 & 0 & 0 & 0 & 0 & 0 \\
$c$ & 0 & $c$ & $c$ & $c$ & $c$ \\
$a$ & 0 & $c$ & $a$ & $c$ & $a$ \\
$b$ & 0 & $c$ & $c$ & $b$ & $b$ \\
1 & 0 & $c$ & $a$ & $b$ & 1
\end{tabular}

\begin{tabular}{c|ccccc}
$\rightarrow$ & 0 & $c$ & $a$ & $b$ & 1 \\
\hline 0 & 1 & 1 & 1 & 1 & 1 \\
$c$ & 0 & 1 & 1 & 1 & 1 \\
$a$ & 0 & $b$ & 1 & $b$ & 1 \\
$b$ & 0 & $a$ & $a$ & 1 & 1 \\
1 & 0 & $c$ & $a$ & $b$ & 1
\end{tabular}

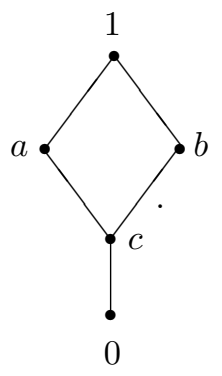

Clearly, $(A, \wedge, \vee, *, \rightarrow, 0,1)$ is an MTL-algebra.

\begin{tabular}{|c|c|c|c|c|c|c|c|c|c|c|c|}
\hline$x$ & 0 & $c$ & $a$ & $b$ & 1 & $x$ & 0 & & $a$ & & \\
\hline$\forall_{1} x$ & 0 & 0 & 0 & $\Omega$ & 1 & $\exists_{1} x$ & D & & 1 & & \\
\hline$\forall_{2} x$ & 0 & $c$ & $c$ & $c$ & 1 & $\exists_{2} x$ & 0 & & 1 & & \\
\hline$\forall{ }_{3} x$ & 0 & $c$ & $a$ & $b$ & 1 & $\exists_{3} x$ & 0 & $c$ & $a$ & & \\
\hline
\end{tabular}


Then the structures $\left(A, \forall_{i}, \exists_{i}\right), i=1,2,3$ are monadic MTL-algebras.

Lemma 3.1. [3] Let $(A, \forall)$ be a monadic $M T L$-algebra. Then the following properties hold, for all $x, y \in A$ :
(1) $\forall \exists x=\exists x$.
(2) $x \leq \exists x$.

115

(3) $\forall 0=0$ and $\forall 1=1$.

(4) $\exists 0=0$ and $\exists 1=1$.

(5) $\forall(x \rightarrow \exists y)=\exists x \rightarrow \exists y$.

(6) $\exists \forall x=\forall x$.

(7) $\exists \exists x=\exists x$ and $\forall \forall x=\forall x$.

(8) $\forall(\exists x \rightarrow \exists y)=\exists x \rightarrow \exists y$.

(9) $\forall(\exists x \rightarrow \exists y)=\exists x \rightarrow \exists y$.

(10) $\exists(x \rightarrow \exists y) \rightarrow(\forall x \rightarrow \exists y)=1$.

(11) $\exists(\exists x * \exists y)=\exists x * \exists y$.

(12) $\exists(x \vee y)=\exists x \vee \exists y$.

120

(13) $\forall x=x$ iff $\exists x=x$.

(14) $\forall(x \wedge y)=\forall x \wedge \forall y$.

(15) $\forall(\exists x \rightarrow b)=\exists x \rightarrow \forall y$.

(16) $\forall(\forall x \vee y)=\forall x \vee \forall y$.

(17) $\exists(\exists x \rightarrow b) \rightarrow(\exists x \rightarrow \exists y)=1$.

(18) $\forall(\exists x \vee \exists y)=\exists a \vee \exists y)$.

(19) $\forall(x \rightarrow y) \rightarrow(\forall x \rightarrow \forall y)=1$.

(20) $\forall(x \rightarrow y) \rightarrow(\exists x \rightarrow \exists y)=1$.

(21) $(\forall x * \exists y) \rightarrow \exists(x * y)=1$.

(21) $(\forall x * \forall y) \rightarrow \exists(x * y)=1$.

125

(22) $\exists(a * \exists y)=\exists x * \exists y$.

(23) $\exists(x * \forall y)=\exists x * \forall y$.

(24) $\exists(\exists x \rightarrow \exists y)=\exists x \rightarrow \exists y$.

(25) $\exists(\forall x \rightarrow \forall y)=\forall x \rightarrow \forall y$.

(26) $\exists(\exists x \wedge \exists y)=\exists x \wedge \exists y$.

(27) $\exists(x \wedge \exists y)=\exists x \wedge \exists y$.

(28) $\forall(\forall x \rightarrow \forall y)=\forall x \rightarrow \forall y$.

(29) $\exists(\forall x * \forall y)=\forall x * \forall y$.

(30) $\forall(\forall x * \forall y)=\forall x * \forall y$.

(31) $\forall(\forall x \wedge \forall y)=\forall x \wedge \forall y$.

Remark 3.1. The operations $\forall$ and $\exists$ are order preserving, i.e. if $x \leq y$, then $\forall x \leq \forall y$ and $\exists x \leq \exists y$.

Proof. Let $x \leq y$. Then $\forall x \leq x \leq y \leq \exists y$. By $\left(M_{3}\right)$, we have $\forall x \rightarrow \forall y=\forall(\forall x \rightarrow y)=\forall 1=1$. From Lemma $3.1(5), \exists x \rightarrow \exists y=\forall(x \rightarrow \exists y)=\forall 1=1$.

Theorem 3.1. Let $(A, \forall, \exists)$ be a monadic MTL-algebra. Then the following conditions hold:
(i) $\neg(\exists x)=\forall(\neg x)$,
(ii) $\exists x \leq \neg \neg(\exists x)=\neg(\forall(\neg x))$, 
(iii) $\forall x \leq \neg(\exists(\neg x))=\forall(\neg \neg x)$,

(iv) $\neg \neg(\forall(\neg \neg x))=\forall(\neg \neg x) \geq \neg \neg(\forall x)$,

(v) $\neg \neg(\exists(\neg \neg x))=\exists(\neg \neg x) \geq \neg \neg(\exists x)$.

Proof. (i) From Lemma 3.1 (4), (5), we have:

$$
\begin{aligned}
\neg(\exists x) & =\exists x \rightarrow 0 \\
& =\exists x \rightarrow \exists 0 \\
& =\forall(x \rightarrow \exists 0) \\
& =\forall(x \rightarrow 0) \\
& =\forall(\neg x) .
\end{aligned}
$$

(ii) By Lemma 2.1, $\exists x \leq \neg \neg(\exists x)$, from $(i)$ we have $\neg \neg(\exists x)=\neg(\forall(\neg x))$.

(iii) From Lemma $3.1(4)$, (5), we get $\neg(\exists(\neg x))=\exists(x \rightarrow 0) \rightarrow 0=\exists(x \rightarrow 0) \rightarrow \exists 0=\forall((x \rightarrow$ $0) \rightarrow 0)=\forall(\neg \neg x)$. By Remark 3.1, $\forall x \leq \neg(\exists(\neg x))=\forall(\neg \neg x)$.

(iv) From (ii), $\exists(\neg x) \leq \neg(\forall(\neg \neg x))$ so $\neg(\exists(\neg x)) \geq \neg \neg(\forall(\neg \neg x))$ and by (iii), $\neg(\exists(\neg x))=$ $\forall(\neg \neg x)$. Thus $\forall(\neg \neg x) \geq \neg \neg(\forall(\neg \neg x))$ and so $\neg \neg(\forall(\neg \neg x))=\forall(\neg \neg x) \geq \neg \neg(\forall x)$.

(v) Using $($ i $),($ ii $),($ iii $)$ and Lemma 3.1 (10), we obtain $\neg \neg(\exists(\neg \neg x))=\neg(\forall(\neg \neg \neg x))=\neg(\forall(\neg x))=$ $\neg \neg(\exists x)$. Also, $\exists(\neg \neg x)=\exists((x \rightarrow 0) \rightarrow 0)=\exists((x \rightarrow 0) \rightarrow \exists 0) \leq \forall(x \rightarrow 0) \rightarrow \exists 0=\neg(\forall(\neg x))=$ $\neg \neg(\exists x)$. Therefore $\neg \neg(\exists(\neg \neg x))=\exists(\neg \neg x) \geq \neg \neg(\exists x)$.

For monadic MTL-algebra $(A, \exists, \forall)$, denote:

$$
\begin{aligned}
& A_{\forall}=\{x \in A \mid x=\forall x\}, \\
& A_{\exists}=\{x \in A \mid x=\exists x\} .
\end{aligned}
$$

Put, $A_{\forall \exists}=A_{\forall} \cap A_{\exists}$.

Example 3.2. In Example 3.1, we have $A_{\forall_{2}}=\{0, c, 1\}, A_{\exists_{1}}=\{0,1\}$. Thus $A_{\forall_{2} \exists_{1}}=\{0,1\}$.

As a consequence of Lemma 3.1, we find:

Proposition 3.1. Let $(A, \exists, \forall)$ be a monadic $M T L$-algebra. Then $A_{\forall}=\forall(A)=\exists(A)=\exists\left(A_{\forall}\right)=$ 155 $\forall\left(A_{\forall}\right)$, where $\forall(A)=\{\forall x \mid x \in A\}$ and $\exists(A)=\{\exists x \mid x \in A\}$. 
Proposition 3.2. Let $(A, \exists, \forall)$ be a monadic MTL-algebra. Then $A_{\forall \exists}$ is a subalgebra of $M T L$ algebra A.

Proof. Clearly, $A_{\forall \exists} \neq \emptyset$. For all $x, y \in A_{\forall \exists}$, by Lemma 3.1 (11), (12), (13) we have $\exists(x * y)=$ $\exists(\exists x * y)=\exists x * \exists y=x * y$ and $\exists(x \vee y)=\exists x \vee \exists y=x \vee y, \exists(x \wedge y)=x \wedge y$.

Also, from Lemma 3.1(8), $\forall(\exists x \rightarrow \exists y)=\exists x \rightarrow \exists y$ thus by Lemma $3.1(10), \exists(\exists x \rightarrow \exists y)=$ $\exists x \rightarrow \exists y$ and so $\exists(x \rightarrow y)=x \rightarrow y$. Moreover, $\exists 0=0, \exists 1=1$.

Proposition 3.3. Let $(A, \exists, \forall)$ be a monadic MTL-algebra. Then the following identities hold, for every $x, y \in A$ :

(i) $\exists(x \rightarrow y) \leq \forall x \rightarrow \exists y$.

(ii) $\forall(\forall x \rightarrow y)=\forall x \rightarrow \forall y$.

Proof. (i) Let $x$ and $y$ be in $A$. Because $\forall x$ and $\exists y$ are in $A_{\forall}$, also $\forall x \rightarrow \exists y \in A_{\forall}$. So $\forall x \rightarrow \exists y=$ $\exists(\forall x \rightarrow \exists y)(C)$. Using $\left(M_{1}\right)$ and Lemma 3.1(2), $\forall x \leq x, y \leq \exists y$, we find $x \rightarrow y \leq \forall x \rightarrow \exists y(M)$. Thus, $\exists(x \rightarrow y) \leq \forall x \rightarrow \exists y(M)$.

(ii) By $\left(M_{1}\right)$ and $(M)$ we have $\forall x \rightarrow \forall y \leq \forall x \rightarrow y$, so $\forall x \rightarrow \forall y=\forall(\forall x \rightarrow \forall y) \leq \forall(\forall x \rightarrow y)$ by $(C)$ and $(M)$. On the other hand, by $\left(M_{1}\right), \forall(\forall x \rightarrow y) \leq \forall x \rightarrow y$. By the residuation principle, $\forall x * \forall(\forall x \rightarrow y) \leq y$. Moreover, $\forall x * \forall(\forall x \rightarrow y)=\forall(\forall x * \forall(\forall x \rightarrow y)) \leq \forall y$, by $(C)$ and $(M)$. Applying the residuation principle again, we obtain $\forall(\forall x \rightarrow y)=\forall x \rightarrow \forall y$.

Definition 3.2. Let $(A, \exists, \forall)$ be a monadic MTL-algebra. A monadic filter $F$ of $A$ is a non-empty subset $F$ of $A$ satisfying:

(F.1) if $x \in F, y \in A$ and $x \leq y$, then $y \in F$,

(F.2) if $x, y \in F$, then $x * y \in F$,

(F.3) if $x \in F$, then $\forall x \in F$.

Example 3.3. Consider Example 3.1, $F=\{b, 1\}$ is a monadic filter of $\left(A, \forall_{3}, \exists_{2}\right)$.

Definition 3.3. For a nonempty subset $S \subseteq A$, the smallest monadic filter of $A$ which contains $S$, i.e. $\cap\{F: S \subseteq F\}$, is said to be the monadic filter of $A$ generated by $S$ and will be denoted by $[S)$. If $S=\{a\}$, with $a \in A$, we denoted by $[a)$ the monadic filter generated by $\{a\}$ ([a) is called principal). 
We will denote the set of all monadic filters of $(A, \forall, \exists)$ by $M F(A)$.

185

Proposition 3.4. Let $S \subseteq A$, a nonempty subset of $A, a \in A$. Then

In particular, $[a)=\left\{x \in A:(\forall a)^{n} \leq x\right.$, for some $\left.n \geq 1\right\}$.

Proof. Let $\mathrm{M}=\left\{x \in A: \forall s_{1} * \ldots * \forall s_{n} \leq x\right.$, for some $n \geq 1$ and $\left.s_{1}, \ldots, s_{n} \in S\right\}$. Then $M$ is a monadic filter which contain the set $S$, hence $[S) \subseteq M$. Let $F$ be a monadic filter such that $S \subseteq F$ and $x \in M$. Then there exist $s_{1}, s_{2}, \ldots, s_{n} \in S$ such that $\forall s_{1} * \ldots * \forall s_{n} \leq x$. Since $s_{1}, s_{2}, \ldots, s_{n} \in F$, $\forall s_{1} * \ldots * \forall s_{n} \in F, x \in F$. Hence $M \subseteq F$, therefore $M \subseteq F=[S)$, that is, $[S)=M$.

If $F$ is a monadic filter and $x \notin F$, put $[F, x):=[F \cup\{x\})$.

Theorem 3.2. Let $F, F_{1}, F_{2}$ be monadic filters of $A$ and $a \notin F$. Then

(i) $[F \cup a)=\left\{x \in A \mid x \geq f *(\forall a)^{n}, f \in F\right\}=F \vee[\forall a)$,

(ii) $\left[F_{1} \cup F_{2}\right)=\left\{x \in A \mid x \geq f_{1} * f_{2}, f_{1} \in F_{1}, f_{2} \in F_{2}\right\}$,

(iii) if $a \leq b$, then $[b] \subseteq[a)$,

(iv) $[\forall a)=[a)$,

$(v)[a) \vee[b)=[a \wedge b)=[a * b)$,

(vi) $[a) \cap[b)=[\forall a \vee \forall b)$. deduce that $t \geq(\forall)^{m}$ and $t \geq(\forall b)^{n}$. Hence $t \geq(\forall a)^{m} \vee(\forall b)^{n} \geq(\forall a \vee \forall b)^{n m}=(\forall(a \vee b))^{n m}$ and $a \in[\forall a \vee \forall b)$, that is $[a) \cap[b] \subseteq[\forall a \vee \forall b)$. Therefore $[a) \cap[b]=[\forall a \vee \forall b)$.. 
Theorem 3.3. Let $F$ be a monadic filter of $(A, \forall, \exists)$. Then the following conditions are equivalent: $x \in A$. It is clear that $\forall_{F}$ and $\exists_{F}$ is well defined.

Clearly, the following proposition hold:

Proposition 3.6. Let $F$ be a monadic filter of $(A, \forall, \exists)$. Then $\left(A / F, \forall_{F}, \exists_{F}\right)$ is a monadic $M T L$ algebra.

(i) $F$ is a monadic filter of $(A, \forall, \exists)$,

(ii) $F=\left[F \cap A_{\forall}\right)$.

Proposition 3.5. Let $(A, \forall, \exists)$ be a monadic MTL-algebra, $x \in A_{\forall}$ and $F$ be a monadic filter of $(A, \forall, \exists)$. Then $[F \cup\{x\})$ is also a monadic filter of $(A, \forall, \exists)$.

Definition 3.4. Let $(A, \forall, \exists)$ be a monadic $M T L$-algebra and $\theta$ be a congruence on $A$. Then $\theta$ is called a monadic congruence on $(A, \forall, \exists)$ if $(x, y) \in \theta$ implies $(\forall x, \forall y) \in \theta$, for all $x, y \in A$. Where:

$$
(x, y) \in \theta \text { iff }(x \rightarrow y) \wedge(y \rightarrow x) \in F .
$$

Theorem 3.4. For all monadic MTL-algebra there exists a one to one correspondence between its monadic filters and its monadic congruences.

Let $(A, \forall, \exists)$ be a monadic MTL-algebra and $F$ be a monadic filter. We define a mapping $\forall_{F}: L / F \rightarrow L / F$ such that $\forall_{F}([x])=[\forall x]$ and $\exists_{F}: L / F \rightarrow L / F$ such that $\exists_{F}([x])=[\exists x]$, for all

Definition 3.5. A proper monadic filter $F$ of $(A, \forall, \exists)$ is called a prime monadic filter of $(A, \forall, \exists)$, if $F_{1} \cap F_{2} \subseteq F$, implies $F_{1} \subseteq F$ or $F_{2} \subseteq F$, for all $F_{1}, F_{2} \in M F(A)$.

Example 3.4. In Example 3.1, $F=\{c, a, b, 1\}$ is a prime monadic filter of monadic MTL-algebra $\left(A, \forall_{2}, \exists_{2}\right)$.

Theorem 3.5. Let $F$ be a monadic filter of $(A, \forall, \exists)$. Then the following are equivalent:

(i) $F$ is a prime monadic filter of $(A, \forall, \exists)$,

(ii) if $\forall x \vee \forall y \in F$, then $\forall x \in F$ or $\forall y \in F$, for some $x, y \in A$,

(iii) $\forall x \rightarrow \forall y \in F$ or $\forall y \rightarrow \forall x \in F$, for any $x, y \in A$,

(iv) $\left(A / F, \forall_{F}, \exists_{F}\right)$ is a linearly ordered monadic $M T L$-algebra.

Proof. $(i \Rightarrow$ ii) Let $\forall x \vee \forall y \in F$, for some $x, y \in A$. Then $[x) \cap[y)=[\forall x \vee \forall y) \in F$. Since $F$ is a prime monadic filter of $(A, \forall, \exists)$, then $[x) \subseteq F$ or $[y) \subseteq F$. So $\forall x \in F$ or $\forall y \in F$. 
$(i i \Rightarrow i i i)$ Since $(\forall x \rightarrow \forall y) \vee(\forall y \rightarrow \forall x)=1 \in F$, we have $\forall x \rightarrow \forall y \in F$ or $\forall y \rightarrow \forall x \in F$.

$(i i i \Rightarrow i v)$ It follows from Proposition 3.6, that $\left(A / F, \forall_{F}, \exists_{F}\right)$ is a monadic MTL-algebra. Now, we prove that is linearly ordered. If $[x],[y] \in A / F$, from $(i i i)$, then $[x] \leq[y]$ or $[y] \leq[x]$. Thus $\left(A / F, \forall_{F}, \exists_{F}\right)$ is a linearly ordered monadic MTL-algebra.

$(i v \Rightarrow i)$ It is clear.

Let $(A, \forall, \exists)$ be a monadic MTL-algebra. Denote:

$$
\begin{aligned}
& \operatorname{Ker}(\forall)=\{x \in A \mid \forall x=1\} . \\
& \operatorname{Ker}(\exists)=\{x \in A \mid \exists x=1\} .
\end{aligned}
$$

245

Definition 3.6. A proper monadic filter $F$ of $(A, \forall, \exists)$ is calt
strictly contained in any proper monadic filter of $(A, \forall, \exists)$.

Example 3.5. In Example 3.1, $F=\{c, a, b, 1\}$ is a maximal monadic filter of $\left(A, \forall_{2}, \exists_{1}\right)$. Clearly, we have $\operatorname{Ker}\left(\forall_{1}\right)=\{1\}$.

Theorem 3.6. Let $F$ be a proper monadic filter of $(A, \forall, \exists)$. Then the following conditions are

250 equivatent:

(i) $F$ is a maximal monadic filter of $(A, \forall, \exists)$.

(ii) $\forall x \in F$ or $\neg \forall x \in F$, for all $x \in A$,

(iii) $\exists x \in F$ or $\neg \exists x \in F$, for all $x \in A$.

Proof. $(i \Rightarrow i i)$ Let $F$ be a maximal monadic filter and there exists $x \in A$ such that $\forall x \notin F$ and 255 $\neg \forall x \notin F$. As $\neg \forall x \notin F$, we have $[\forall x \cup F)$ is proper. By Proposition 3.5, we obtain $[F \cup\{x\})$ is also a monadic filter of $(A, \forall, \exists)$. According to our assumption, $F$ is strictly contained in $[\forall x \cup F)$ which leads to a contradiction.

$(i i \Rightarrow i)$ Let $(i i)$ and in contrary to $(i), F$ is not a maximal monadic filter. Then there exists a proper monadic filter $M$ strongly containing $F$. But there exists $x \in M$ such that $x \notin F$. So $\forall x \notin F$. By our assumption, $\neg \forall x \in F$. Hence, $\neg \forall x \in M$. Therefore, as $x \in M$ and $M$ is a monadic filter, $\forall x \in M$. But this contradicts the fact that $M$ is a proper filter.

$(i i \Leftrightarrow i i i)$ It is clear. 
Theorem 3.7. Let $F$ be a monadic filter of monadic MTL-algebra $(A, \forall, \exists)$ and a $\notin F$. Then there exists a prime monadic filter $P$ of $(A, \forall, \exists)$ such that $F \subseteq P$ and $a \notin P$.

Proof. $(i \Rightarrow i i)$ Let $F$ be a filter of $A_{\forall}$ and $F \neq\{1\}$. Consider

$$
F_{f}=\{t \in A \mid t \geq f \text { for a certain } f \in F\} .
$$

Then there exists $f_{1}, f_{2} \in F$ such that $x \geq f_{1}, y \geq f_{2}$, for $x, y \in F_{f}$. So $x * y \geq f_{1} * f_{2} \in F$, hence $x * y \in F_{f}$. If $x \in F_{f}, x \leq y$, then $y \in F_{f}$, and if $x \in F_{f}$, then $x \geq f, f \in F$. Since $f \in A_{\forall}$, thus 

$F_{f} \neq\{1\}$, it follows that $F_{f}=A$. Hence $0 \in F_{f}$ and $0 \in F$, that is $F=\forall A$.

$(i i \Rightarrow i)$ Let $F$ be a monadic filter of $(A, \forall, \exists)$. Then $F \cap A_{\forall}$ is a filter of $A_{\forall}$, so $F \cap A_{\forall}=\{1\}$ or $F \cap A_{\forall}=A_{\forall}$. If $F \cap A_{\forall}=A_{\forall}$, then $A_{\forall} \subseteq F$. Since $0 \in A_{\forall}$, then $F=A$. Since $\operatorname{Ker}(\forall)=\{1\}$, if $F \cap A_{\forall}=\{1\}$ and $x \in F$, then $\forall x \in F \cap A_{\forall}$, so $\forall x=1$, that is $x=1$ so $F=\{1\}$. Thus $(A, \forall, \exists)$

Let $(A, \forall, \exists)$ be subdirectly irreducible monadic MTL-algebra. Then by Theorem 3.4, there exists a monadic filter $F$ of $(A, \forall, \exists)$ such that $\theta_{F}=F$. Thus, we can conclude that a monadic $\operatorname{MTL}$-algebra $(A, \forall, \exists)$ is said to be subdirectly irreducible if among the nontrivial monadic filters of $(A, \forall, \exists)$ there exists the least one, i.e., $\cap\{F \in M F(A) \mid F \neq\{1\}\} \neq\{1\}$.

Proposition 3.7. Let $(A, \forall, \exists)$ be a subdirectly irreducible monadic $M T L$-algebra and $F_{1}, F_{2} \in$ $M F(A)$. If $F_{1} \cap F_{2}=\{1\}$, then $F_{1}=\{1\}$ or $F_{2}=\{1\}$.

Proof. Let $F_{1} \neq\{1\}$ and $F_{2} \neq\{1\}$, i.e. $F_{1}, F_{2} \in \cap\{F \in M F(A) \mid F \neq\{1\}\} \neq\{1\}$. Then $\cap\{F \in$ $M F(A) \mid F \neq\{1\}\} \neq\{1\} \subseteq F_{1} \cap F_{2}$. By $F_{1} \cap F_{2}=\{1\}$, we have $\cap\{F \in M F(A) \mid F \neq\{1\}\}=\{1\}$, which contradictions to the fact that $(A, \forall, \exists)$ is a subdirectly irreducible monadic MTL-algebra. So $F_{1}=\{1\}$ or $F_{2}=\{1\}$.

\section{Theorem 3.9. The following conditions are equivalent:}

(i) $(A, \forall, \exists)$ is a subdirectly irreducible monadic MTL-algebra,

(ii) there exists an element $a \in A, a<1$, such that for all $x \in A, x<1$ and $a \in[x)$.

Proof. $(i \Rightarrow i i)$ If $(A, \forall, \exists)$ is a subdirectly irreducible monadic MTL-algebra, i.e.,$\cap\{F \in M F(A) \mid$ $F \neq\{1\}\} \neq\{1\}$, then $\cap\{[x) \mid x<1\} \neq\{1\}$. Suppose that $a \in \cap\{[x) \mid x<1\}$ satisfying $a \neq 1$. 315 Then for all $x \in A, x \neq 1$ and $a \in[x)$. By Theorem 3.2, there exists $m \in \mathbb{N}$, such that $a \geq(\forall x)^{m}$. Clearly, $a$ is the element that we need. 
(ii $\Rightarrow i$ ) We show that for any $F \in M F(A)$, if $F \neq\{1\}$, then $a \in F$. Since $F \neq\{1\}$, there exists $x \in F, x<1$, then by the know condition, $a \in[x)$ and $a \in F$. Hence $a \in\{F \in M F(A) \mid F \neq\{1\}\}$. Thus $\cap\{F \in M F(A) \mid F \neq\{1\}\} \neq\{1\}$, i.e. $(A, \forall, \exists)$ is a subdirectly irreducible monadic MTLalgebra.

Proposition 3.8. Let $(A, \forall, \exists)$ be a subdirectly irreducible monadic MTL-algebras. If $x \vee y=1$, then $x=1$ or $y=1$, for all $x, y \in A$.

Proof. If $x \vee y \in A$, then by Theorem 3.2, we have $[x) \cap[y)=[\forall x \vee \forall y)=[\forall(x \vee y))=[1]=\{1\}$. By Proposition 3.7, we have $[x)=\{1\}$ or $[y)=\{1\}$. So $x=1$ or $y=1$.

Proposition 3.9. Let $(A, \exists, \forall)$ be a subdirectly irreducible monadic MTL-algebra. Then $(A, \exists, \forall)$ is a chain.

Proof. Suppose that $(A, \exists, \forall)$ is a subdirectly irreducible monadic MTL-algebra. Since $(x \rightarrow y) \vee$ $(y \rightarrow x)=1$, by Proposition 3.8, we have $x \rightarrow y=1$ or $y \rightarrow x=1$, i.e. $x \leq y$ or $y \leq x$. So $(A, \exists, \forall)$ is a chain.

Proposition 3.10. If $(A, \exists, \forall)$ is a subdirectly irreducible monadic $M T L$-algebra, then $A_{\forall}$ is linearly ordered.

Proof. Let $F$ be the smallest non-trivial monadic filter of $(A, \exists, \forall)$ and $x \in F \backslash\{1\}$. If $A_{\forall}$ is not linearly ordered, and $\forall a, \forall b \in A_{\forall}$ such that $\forall a \not \leq \forall b$ and $\forall b \not \leq \forall a$. Then the monadic filters $[\forall a \rightarrow$ $\forall b)$ and $[\forall b \rightarrow \forall a)$ generated by $\forall a \rightarrow \forall b$ and $\forall b \rightarrow \forall a$ respectively are non-trivial. Thus both contain $F$, in particular $x \in[\forall a \rightarrow \forall b)$ and $x \in[\forall b \rightarrow \forall a)$. Since $\forall a \rightarrow \forall b \in A_{\forall}$, by Theorem 3.2, there is an $n$ such that $x \geq(\forall(\forall a \rightarrow \forall b))^{n} \geq(\forall a \rightarrow \forall b)^{n}$ and $x \geq(\forall(\forall b \rightarrow \forall a))^{n} \geq(\forall b \rightarrow \forall a)^{n}$. By Proposition 2.1, $x \geq(\forall a \rightarrow \forall b)^{n} \vee(\forall b \rightarrow \forall a)^{n}$. Hence $x=1$ which is a contradiction.

Theorem 3.10. $(A, \forall, \exists)$ is a subdirect irreducible monadic MTL-algebra iff $A_{\forall}$ is a subdirectly irreducible subalgebra of $A$.

Proof. Let $(A, \forall, \exists)$ be a subdirect irreducible monadic MTL-algebra. Then $M F(A) \backslash\{1\}$ has a minimal element $F$. Clearly, $A_{\forall}$ is a subalgebra of $A$. We show that $F \cap A_{\forall}$ is the minimal monadic filter of $A_{\forall}$ such that $F \cap A_{\forall} \neq\{1\}$. If $F \cap A_{\forall}=\{1\}$, since $\forall F \subseteq F \cap A_{\forall}$ then $\forall x=1$, for all $x \in F$. Hence $F \subseteq \operatorname{Ker}(\forall)=\{1\}$ and $F=\{1\}$, which is a contradiction. That means $F \cap A_{\forall} \neq\{1\}$. 
345

Now, we show that $F \cap A_{\forall}$ is the minimal monadic filter of $(A, \forall, \exists)$. Suppose that $G$ is a filter of $A_{\forall}$ and hence $[G)$ is the monadic filter of $(A, \forall, \exists)$ generated by $G$. Clearly, $[G) \cap A_{\forall}=G$. By minimality of $F, F \subseteq[G)$ and so $F \cap A_{\forall} \subseteq[G) \cap A_{\forall}$. Thus $F \cap A_{\forall}$ is the minimal filter of $A_{\forall}$ such that $F \cap A_{\forall} \neq\{1\}$. Then $A_{\forall}$ is a subdirectly irreducible subalgebra of $A$.

Conversely, $A_{\forall}$ is a subdirectly irreducible subalgebra of $A$. Then there is a minimal filter $F$ of $A_{\forall}$ such that $F \neq\{1\}$. From Theorem 3.3, $\left[F \cap A_{\forall}\right)$ is a monadic filter of $(A, \forall, \exists)$. We show that $\left[F \cap A_{\forall}\right)$ is a minimal monadic filter of $(A, \forall, \exists)$. In fact, if $G$ is another non-trivial monadic filter of $(A, \forall, \exists)$, then $G \cap A_{\forall} \supseteq F \cap A_{\forall}$. Then $G$ contains the monadic filter generated by $F$, that is, $\left[F \cap A_{\forall}\right) \subseteq\left[G \cap A_{\forall}\right)$, i.e., $\left[F \cap A_{\forall}\right)$ is minimal. Thus, $(A, \forall, \exists)$ is a subdirectly irreducible monadic MTL-algebra.

Proposition 3.11. Let $F$ be a monadic filter of $(A, \forall, \exists)$. Then $F=[F \cup\{x \rightarrow y\}) \cap[F \cup\{y \rightarrow x\})$, for all $x, y, z \in A$.

Proof. Let $z$ be an element of both $[F \cup\{x \rightarrow y\})$ and $[F \cup\{y \rightarrow x\})$. Then, there are $f_{1}, f_{2} \in$ $F, n_{1}, n_{2} \in \mathbb{N}$ such that $f_{1} *\left(\forall(x \rightarrow y)^{n_{1}} \leq z\right.$ and $f_{2} *\left(\forall(y \rightarrow x)^{n_{2}} \leq z\right.$. If $f=f_{1} * f_{2}$ and $n=\max \left\{n_{1}, n_{2}\right\}$, so $f *(\forall(x \rightarrow y))^{n} \leq z$ and $f *(\forall(y \rightarrow x))^{n} \leq z$. Thus $(\forall(x \rightarrow y))^{n} \leq f \rightarrow z$ and $(\forall(y \rightarrow x))^{n} \leq f \rightarrow z$ and so $(\forall(x \rightarrow y))^{n} \vee(\forall(y \rightarrow x))^{n} \leq f \rightarrow z$. But $(\forall(x \rightarrow y))^{n} \vee(\forall(y \rightarrow$ $x))^{n}=(\forall((x \rightarrow y) \vee(y \rightarrow x)))^{n}=\forall 1=1$, so $f \leq z$ and $z \in F$. The forward inclusion is straightforward.

Proposition 3.12. There exists a prime monadic filter $P$ of $(A, \forall, \exists)$, such that a $\vee \forall r \notin P$, for all $a, r \in A, a, r \neq 1$.

Proof. Let $C=\{a \vee \forall r \mid r \neq 1\}$. Note that $1 \notin C$, Since $a \vee \forall r=1$ then we have $1=$ $\forall(a \vee \forall r)=\forall a \vee \forall r$, and so $a=1$ or $r=1$. Let $\mathcal{F}$ be the family of monadic filters $F$ in $(A, \forall, \exists)$ such that $F \cap C=\emptyset$. Clearly, $1 \in \mathcal{F}$, so $\mathcal{F} \neq \emptyset$. It is straightforward to verify that any chain in $\mathcal{F}$ has an upper bound in $\mathcal{F}$. By Lemma Zorn's, there exists a maximal filter $P$ in $\mathcal{F}$. We show that $P$ is prime monadic filter. We claim that $P$ is prime monadic filter. Note that $P=[P \cup\{x \rightarrow y\}) \cap[P \cup\{y \rightarrow x\})$. If we assume that neither $P=[P \cup\{x \rightarrow y\})$ nor $P=\left[P \cup\{y \rightarrow x\}\right.$ ) belongs to $\mathcal{F}$, then there are $r_{1}, r_{2} \neq 1$ such that $a \vee \forall r_{1} \in P=[P \cup\{x \rightarrow y\})$ and $a \vee \forall r_{2} \in P=[P \cup\{y \rightarrow x\})$. Since $\forall r_{1}$ and $\forall r_{2}$ are comparable, it follows that one of them belongs to both filters. Hence one of them belongs to $P$, which is a contradiction. This shows that either $[P \cup\{x \rightarrow y\}) \in \mathcal{F}$ or $[P \cup\{y \rightarrow x\}) \in \mathcal{F}$. By the maximality of $P$, we have $P=[P \cup\{x \rightarrow y\})$ and so $x \rightarrow y \in P$. Similarly, If $P=[P \cup\{y \rightarrow x\})$, then $y \rightarrow x \in P$. 
Theorem 3.11. Let $(A, \forall, \exists)$ be a monadic $M T L$-algebra. Then there exists a subdirect representation of the monadic $M T L$-algebra $A \leq \prod_{i \in I} A_{i}$, where $A_{\forall}$ is embedded in $A_{i}$ via the corresponding projection map.

Proof. Let $P_{a}$ be one of the prime monadic filters provided by the Proposition 3.12, for all $a \in$

$\forall q) \leq \exists p \rightarrow \forall q$ and $\forall(p \rightarrow \forall q)=\exists p \rightarrow \forall q$. So $\left(M_{2}\right)$ hold. 
$\left(M_{3}\right)$ By assumption, we have $\forall(\forall p \rightarrow q)=\bigwedge_{b \in R(q)}(\bigwedge R(p) \rightarrow b)$ and $\forall p \rightarrow \forall q=\bigwedge R(p) \rightarrow$ $\bigwedge R(q)$. For all $b \in R(q), \bigwedge R(q) \leq b$. By Lemma $2.1, \wedge R(p) \rightarrow \wedge R(q) \leq \wedge R(p) \rightarrow b$. So

$\bigwedge R(p) \rightarrow \bigwedge R(q) \leq \bigwedge_{b \in R(q)}(\bigwedge R(p) \rightarrow b)$ and $\forall p \rightarrow \forall q \leq \forall(\forall p \rightarrow q)$.

Conversely, for all $b \in R(q)$, we have $c \leq \wedge R(p) \rightarrow b$. Thus $c * \wedge R(p) \leq b$ and $c * \bigwedge R(p) \leq$ $\bigwedge R(q)$. So $c \leq \bigwedge R(p) \rightarrow \bigwedge R(q)$ and $\bigwedge_{b \in R(q)}(\bigwedge R(p) \rightarrow b) \leq \wedge R(p) \rightarrow \wedge R(q)$. Therefore $\forall(\forall p \rightarrow \forall q) \leq \forall p \rightarrow \forall q$ and $\forall(\forall p \rightarrow \forall q)=\forall p \rightarrow \forall q$. So $\left(M_{3}\right)$ hold.

$\left(M_{4}\right)$ We have $\forall(p \vee \exists q)=\bigwedge R(p \vee \vee R(q))=\bigwedge_{a \in R(p)}\left(a \vee \bigvee_{b \in R(q)} b\right)$. On the other hand, $\forall p \vee \exists q=\bigwedge R(p) \vee \bigvee R(q)=\bigwedge_{a \in R(p)}(a \vee \bigvee R(q))=\bigwedge_{a \in R(p)}\left(a \vee \bigvee_{b \in R(q)} b\right)$. So $\left(M_{4}\right)$ hold.

$\left(M_{5}\right) \exists p * \exists p=\bigvee R(p) * \bigvee R(p)=\bigvee_{a \in R(p)} a * \bigvee_{b \in R(p)} b=\bigvee_{a \in R(p)} \bigvee_{b \in R(p)}(a * b)=\bigvee_{a, b \in R(p)}(a *$ b) $=\exists(p * p)$.

\section{Monadic Monoidal t-norm based logic}

In this section, we develop a propositional calculus, monadic monoidal t-norm based logic, denoted $\mathcal{M M T} \mathcal{L}$, which has monadic MTL-algebra as models.

The language of $\mathcal{M M T} \mathcal{L}$ consists of:

- countably many proposition variables $\left(p_{1}, p_{2}, \ldots\right)$, the constants $\overline{0}$ and $\bar{u}$,

- the unary operators $\square, \diamond$, the binary operators $\sqcup, \sqcap, \&, \rightarrow$,

- a binary logical connective $\Leftrightarrow$,

-the auxiliary symbols ( and ).

The formulas of $\mathcal{M M T} \mathcal{L}$ are defined inductively as follows:

$f_{1}$. proposition variables, $\overline{0}$ is formula,

$f_{2}$. if $\varphi$ and $\psi$ are formulas, then so are $(\varphi \sqcap \psi),(\varphi \sqcup \psi),(\varphi \& \psi),(\varphi \rightarrow \psi),(\varphi \Leftrightarrow \psi),(\square \psi)$ and $(\diamond \psi)$

$f_{3}$. a string of symbols is a formula of $\mathcal{M M T} \mathcal{L}$ iff it can be show to be a formula by a finite number of applications of $f_{1}, f_{2}$.

In order to avoid unnecessary brackets, we agree on the following priority rules:

- unary operators always take precedence over binary ones, while,

- among the binary operators, \& has the highest priority; furthermore $\sqcap$ and $\sqcup$ take precedence 
- the outermost brackets are not written. We also introduce some useful shorthand notations: $\overline{1}$ for $\overline{0} \rightarrow \overline{0}, \neg \varphi$ for $\varphi \rightarrow \overline{0}$, for formulas $\varphi$ and $\psi$.

The axioms of $\mathcal{M M T} \mathcal{L}$ are defined as follows:

I. any axioms of $(\mathcal{M M T} \mathcal{L})$ is an axiom of $\mathcal{M T} \mathcal{L}$, i.e. formula which has one of the following 435 forms is an axiom (where $\varphi, \psi$ and $\chi$ are arbitrary formulas):

$\left(A_{1}\right)(\varphi \rightarrow \psi) \rightarrow((\psi \rightarrow \chi) \rightarrow(\varphi \rightarrow \chi))$

$\left(A_{2}\right)(\varphi \& \psi) \rightarrow \varphi$,

$\left(A_{3}\right)(\varphi \& \psi) \rightarrow(\psi \& \varphi)$,

$\left(A_{4}\right)(\varphi \sqcap \psi) \rightarrow \varphi$,

440

$\left(A_{5}\right)(\varphi \sqcap \psi) \rightarrow(\psi \sqcap \varphi)$,

$\left(A_{6}\right)(\varphi \&(\varphi \rightarrow \psi)) \rightarrow(\varphi \sqcap \psi)$,

$\left(A_{7}\right)(\varphi \rightarrow(\psi \rightarrow \chi)) \rightarrow((\varphi \& \psi) \rightarrow \chi)$,

$\left(A_{8}\right)((\varphi \& \psi) \rightarrow \chi) \rightarrow(\varphi \rightarrow(\psi \rightarrow \chi))$,

$\left(A_{9}\right)((\varphi \rightarrow \psi) \rightarrow \chi) \rightarrow(((\psi \rightarrow \varphi) \rightarrow \chi) \rightarrow \chi)$

445

$\left(A_{10}\right) \overline{0} \rightarrow \varphi$.

II. a formula which has one of the following forms is an axiom (where $\varphi$ and $\psi$ are arbitrary formulas):

$(\square 1) \square \varphi \rightarrow \varphi$,

$(\diamond 1) \varphi \rightarrow \diamond \varphi$,

450

$(\square 2) \square(\psi \rightarrow \varphi) \rightarrow(\psi \rightarrow \square \varphi)$,

$(\diamond 2) \square(\varphi \rightarrow \psi) \rightarrow(\diamond \varphi \rightarrow \psi)$,

$(\square 3) \square(\psi \sqcup \varphi) \rightarrow(\psi \sqcup \square \varphi)$,

$(\diamond 3) \diamond(\varphi \& \varphi) \leftrightarrow \diamond \varphi \& \diamond \varphi$

The deduction rules are modus ponens (MP, from $\varphi$ and $\varphi \rightarrow \psi$ infer $\psi$ ), generalization (G, 455 from $\varphi$ infer $\square \varphi$ ). 
If $\Gamma \subseteq$ Form $_{\mathcal{M M T} \mathcal{L}}$, then a formula $\varphi$ is a $\Gamma$-theorem if there exists a natural number $n \geq 1$ and a sequence of formulas $\varphi_{1}, \ldots, \varphi_{n}=\varphi$ such that, for any $1 \leq i \leq n$ one of the following conditions holds:

$1-\varphi_{i}$ is an axiom,

$2-\varphi_{i} \in \Gamma$,

3- there are $j, k<i$ such that $\varphi_{j}$ is $\varphi_{k} \rightarrow \varphi_{i}$,

The sequence $\varphi_{1}, \ldots, \varphi_{n}=\varphi$ is a $\Gamma$-proof for $\varphi$. The set of $\Gamma$-theorem will be denoted by $\operatorname{Theor}_{\mathcal{M M T} \mathcal{L}}(\Gamma)$. The fact that a formula $\varphi$ is a $\Gamma$-theorem will be simply denoted by $\Gamma \vdash_{\mathcal{M M T} \mathcal{L}} \varphi$. A formula $\varphi$ will be called a theorem if it is provable form the emptyset. This will be denoted by

$\vdash_{\mathcal{M M T L}} \varphi$. In this case, a proof for $\varphi$ will be a sequence of formulas $\varphi_{1}, \ldots, \varphi_{n}=\varphi$ such that for any $i \in\{1, \ldots, n\}$, one of the above conditions 1,3 or 4 is satisfied. The set of all theorems of will be denoted by Theor $\mathcal{M M T \mathcal { L }}$.

Remark 4.1. The propositional calculus $\mathcal{M M T} \mathcal{L}$ is an extension of $\mathcal{M T} \mathcal{L}$. It is straightforward

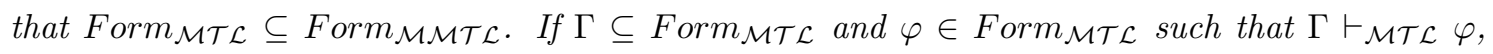
then $\Gamma \vdash_{\mathcal{M M T} \mathcal{L}} \varphi$. Also, every theorem of $\mathcal{M T} \mathcal{L}$ is a theorem of $\mathcal{M M T} \mathcal{L}$.

Definition 4.1. Let $(A, \forall, \exists)$ be a monadic $M T L$-algebra and $\Gamma$ be a theory. An e-evaluation is a mapping e from the set of formulas of $\mathcal{M M T} \mathcal{L}$ to A that satisfies, for all two formulas $\varphi$ and $\psi$ :

$$
\begin{gathered}
e(\varphi \Rightarrow \psi)=e(\varphi) \rightarrow e(\psi), \\
e(\varphi \sqcup \psi)=e(\varphi) \vee e(\psi), \\
e(\varphi \sqcap \psi)=e(\varphi) \wedge e(\psi), \\
e(\varphi \& \psi)=e(\varphi) * e(\psi), \\
e(\square \varphi)=\forall e(\varphi), \\
e(\nabla \varphi)=\exists e(\varphi), \\
e(\overline{0})=0 .
\end{gathered}
$$

If an e-evaluation e satisfies $e(\chi)=1$, for any $\chi$ in $\Gamma$, it is called an $\mathcal{A}$-model of $\Gamma$.

\subsection{Lindenbaum- Tarski algebra of $\mathcal{M M T} \mathcal{L}$}

In the following $\Gamma \subseteq$ Form $_{\mathcal{M M} \mathcal{M} \mathcal{L}}$ is a fixed set of formulas. Let $\varphi$ and $\psi$ be formulas, we define 


$$
\varphi \equiv_{\Gamma} \psi \text { iff } \Gamma \vdash_{\mathcal{S} \mathcal{L} \mathcal{L}} \varphi \rightarrow \psi \text { and } \Gamma \vdash_{\mathcal{S T} \mathcal{L}} \psi \rightarrow \varphi
$$

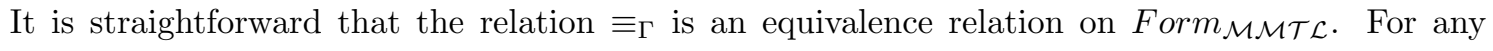

formula $\varphi \in$ Form $_{\mathcal{M M T \mathcal { L }}}$ we will denote by $[\varphi]_{\Gamma}$ the equivalence class of $\varphi$ with respect to $\equiv_{\Gamma}$.

The set Form $_{\mathcal{M M T L} \mathcal{L}} / \equiv_{\Gamma}=\left\{[\varphi]_{\Gamma} \mid \varphi \in\right.$ Form $\left._{\mathcal{A F} \mathcal{L}}\right\}$ is the quotient of $F_{\text {orm }} m_{\mathcal{S T} \mathcal{L}}$ with respect to $\equiv_{\Gamma}$. On $F$ orm $_{\mathcal{S} \mathcal{T} \mathcal{L}} / \equiv_{\Gamma}$ we define the following operations:

This algebras is denoted by $M M T L(\Gamma)=\left(\right.$ Form $\left._{\mathcal{M M} \mathcal{M} \mathcal{L}} / \equiv_{\Gamma}, \vee, \wedge, *, \rightarrow, \forall, \exists, 0,1\right)$.

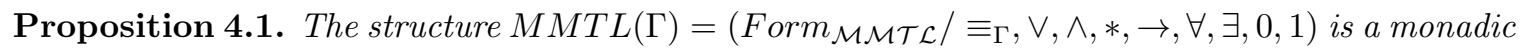
MTL-algebra.

Proof. The proof is similar to that of Proposition 3.6.

Theorem 4.1. $\mathcal{M M T L}$ is complete if and only if the monadic NM-algebra $\left(\right.$ Form $_{\mathcal{M M T} \mathcal{L}} / \equiv_{\Gamma}$ $, \vee, \wedge, *, \rightarrow, \forall, \exists, 0,1)$ is linearly ordered.

Proof. The proof is similar to that of Theorem 3.5.

It is easily verified that $\mathcal{M M T} \mathcal{L}$ is sound with respect to the variety of monadic MTL-algebras, i.e. if a formula $\varphi$ can be deduced from a theory $\Gamma$, then for any $A$-model $e$ of $\mathcal{M M T} \mathcal{L}, e(\varphi)=1$. Indeed, we need to verify the soundness of the new axioms and deductions of $\mathcal{M M T} \mathcal{L}$. For the axioms, it is easy, as they are straightforward generalizations of axioms for monadic MTL-algebras. We will now verify the soundness of the new deduction rules. 
Proposition 4.2. The deduction rules of $\mathcal{M M T L}$ are sound in the following sense, for any formula $\varphi, \psi$ and $A$-model $e$ of $\Gamma$,

(i) If $e(\varphi)=1$, then $e(\forall \varphi)=1$.

(ii) If $e(\varphi)=1$ and $e(\varphi \Rightarrow \psi)=1$, then $e(\psi)=1$.

Proof. (i) If $e(\varphi)=1$, then $e(\forall \varphi)=\forall e(\varphi)=\forall 1=1$ by Lemma 3.1 (3) and Definition 4.1.

(ii) If $e(\varphi)=1$ and $e(\varphi \Rightarrow \psi)=1$, then $1=e(\varphi \Rightarrow \psi)=e(\psi)$ by Lemma 2.1 and Definition 4.1 .

Theorem 4.2. Let $\Gamma$ be a theory over $\mathcal{M M T} \mathcal{L}$ and

varphi be a formula. Then the following statements are equivalent:

(i) $\Gamma \vdash \varphi$,

(ii) for each monadic MTL-algebra $A$ and each A-model e of $\Gamma, e(\varphi)=1$.

\section{Conclusion and future work}

In this work, we have considered the expansion of MTL-algebras with two unary operators $\forall$ and $\exists$. The notion of monadic filters has been introduced and considered them in detail. These types of filters play a basic role and thus the extended filters behave differently. Based on these facts, we give a classification for MTL-algebras. The relationship between monadic MTL-algebras and some related structures is studied. The notion of functional monadic MTL-algebras had been introduced and we show that these structures are particular cases of monadic MTL-algebras. Finally, we introduced monadic monoidal t-norm based logic and proved its completeness based on monadic MTL-algebras. 


\section{Compliance with ethical standards}

\section{References}

[1] M. Baaz, A. Ciabattoni, F. Montagna, Analytic Calculi for Monoidal T-norm Based Logic,

[11] J. T. Wang, X. L. Xin, P. F. He, Monadic NM-algebras, Logic Journal of the IGPL, 27 ( 6), (2019), 812-835.

[12] S. Zahiri, A. Borumand Saeid, E. Eslami, On maximal filters in triangle algebras, Journal of Intelligent and Fuzzy Systems, 30 (2016), 1181-1193. 
[13] S. Zahiri, A. Borumand Saeid, Masoome Zahiri, An investogation on the Co-annihilators in triangle algebras, Iranian Journal of Fuzzy Systems, 15 (7), (2018), 91-102. 